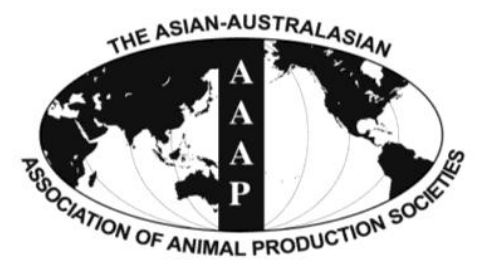

Asian-Aust. J. Anim. Sci.

Vol. 25, No. 6 : 764 - 771

June 2012

www.ajas.info

http://dx.doi.org/10.5713/ajas.2011.11490

\title{
Estimation of Genetic Parameters from Longitudinal Records of Body Weight of Berkshire Pigs
}

\author{
Dong-Hee Lee and Chang-Hee Do* \\ Department of Life Sciences, University of Seoul, 13 Siripdae-kil, Dongdaemun-ku 130-743, Korea
}

\begin{abstract}
Direct and maternal genetic heritabilities and their correlations with body weight at 5 stages in the life span of purebred Berkshire pigs, from birth to harvest, were estimated to scrutinize body weight development with the records for 5,088 purebred Berkshire pigs in a Korean farm, using the REML based on an animal model. Body weights were measured at birth (Birth), at weaning (Weaning: mean $22.9 \mathrm{~d}$ ), at the beginning of a performance test (On: mean $72.7 \mathrm{~d}$ ), at the end of a performance test (Off: mean $152.4 \mathrm{~d}$ ), and at harvest (Finish: mean $174.3 \mathrm{~d}$ ). Ordinary polynomials and Legendre with order 1, 2, and 3 were adopted to adjust body weight with age in the multivariate animal models. Legendre with order 3 fitted best concerning prediction error deviation (PED) and yielded the lowest AIC for multivariate analysis of longitudinal body weights. Direct genetic correlations between body weight at Birth and body weight at Weaning, On, Off, and Finish were 0.48, 0.36, 0.10, and 0.10, respectively. The estimated maternal genetic correlations of body weight at Finish with body weight at Birth, Weaning, On, and Off were 0.39, 0.49, 0.65, and 0.90, respectively. Direct genetic heritabilities progressively increased from birth to harvest and were $0.09,0.11,0.20,0.31$, and 0.43 for body weight at Birth, Weaning, On, Off, and Finish, respectively. Maternal genetic heritabilities generally decreased and were $0.26,0.34,0.15,0.10$, and 0.10 for body weight at Birth, Weaning, On, Off, and Finish, respectively. As pigs age, maternal genetic effects on growth are reduced and pigs begin to rely more on the expression of their own genes. Although maternal genetic effects on body weight may not be large, they are sustained through life. (Key Words: Age, Body Weights, Direct and Maternal Genetic Effects)
\end{abstract}

\section{INTRODUCTION}

Pig productivity traits are analyzed to predict individual breeding values. By using breeding values, high-producing pigs from which progeny are selected as replacements may be identified in seedstock farms. Accurate adjustments need to be made for such factors as parity of the dam, and age at weighing to ensure the most accurate ranking of pigs, since age adjustment is a way to make fairer comparisons between groups with different age distributions. Pigs grow according to a growth curve, in a non-linear manner. The usual growth curve has a sigmoidal-shape with a slow start, an exponential phase and then a plateau phase. Researches on pre-adjustment of age in body weight were carried out by numerous scientists. Bereskin and Norton (1982) calculated multiplicative adjustment factors for adjusting

\footnotetext{
* Corresponding Author: Chang-Hee Do. Department of Animal Biosystem Science, College of Agriculture and Life Science, Chungnam National University, 99 Daehak-ro, Yuseong-gu, Daejeon, Korea. Tel: +82-42-821-5784, Fax: +82-42-823-2766, E-mail: ivando@cnu.ac.kr Submitted Dec. 17, 2011; Accepted Feb. 3, 2012; Revised Mar. 2, 2012
}

pig or litter weight to a $21 \mathrm{~d}$ basis for Duroc and Yorkshire breeds. Quadratic regression equations were fitted to the natural logarithms of pig weights recorded at birth and at 21 and 42 d. Do (2007) adjusted mature body weight by adjustment factors using a growth curve of pigs. It is, however, cumbersome to apply adjustment factors in cases of serial pig weights. Rather using regression in a model with covariate of age is popular in genetic evaluation. Objectives of this study were to examine the schemes of covariates in estimation of genetic parameters and to evaluate the genetic parameters of growth traits of Berkshire pigs.

\section{MATERIAL AND METHODS}

\section{Animals and traits}

Records for 5,088 purebred Berkshire pigs produced from 49 sires mated with 214 dams through 4 generations from 2003 to 2007 in Gyungnam Swine Research Institute (Sancheong, South Korea) were analyzed for this study. Pedigree records for 5,914 animals, including 68 base animals without parent information, were used for the 
Table 1. Number of records according to birth year, gender and sow parity, and numbers of sires and dams

\begin{tabular}{|c|c|c|c|c|c|c|c|c|c|c|}
\hline \multirow{2}{*}{ Birth year } & \multicolumn{3}{|c|}{ Gender } & \multicolumn{5}{|c|}{ Sow parity } & \multicolumn{2}{|c|}{ Parent } \\
\hline & Female & Male & Castrate & 1 & 2 & 3 & 4 & $\geq 5$ & Sire & Dam \\
\hline 2003 & 362 & 343 & 50 & 447 & 308 & 0 & 0 & 0 & 16 & 67 \\
\hline 2004 & 618 & 95 & 549 & 356 & 244 & 320 & 234 & 108 & 22 & 98 \\
\hline 2005 & 817 & 169 & 574 & 316 & 299 & 271 & 198 & 476 & 26 & 117 \\
\hline 2006 & 565 & 450 & 131 & 241 & 161 & 249 & 155 & 340 & 16 & 105 \\
\hline 2007 & 178 & 187 & & 86 & 83 & 65 & 26 & 105 & 12 & 48 \\
\hline Total & 2,540 & 1,244 & 1,304 & 1,446 & 1,095 & 905 & 613 & 1,029 & 92 & 435 \\
\hline Pooled & & 5,088 & & & & 5,088 & & & $49(34)$ & $214(141)$ \\
\hline
\end{tabular}

Note that numbers in the parenthesis represents sire and dams with records.

genetic analysis. Approximately 1,100 pigs, including about 20 sires and 100 dams selected from the herd were maintained through that period. The sires and dams were mated according to a computerized mating plan by using a selection index for genetic improvement in productivity. Details on data structure are presented in Table 1. The pigs had ad libitum access to water and feed (standard scheme), in keeping with the practice in most farms in Korea.

The traits analyzed were individual weights at birth (Birth), at weaning (Weaning), at the beginning of a performance test $(\mathrm{On})$, at the end of the performance test (Off), and at harvest (Finish). Descriptive statistics for the body weights analyzed are presented in Table 2 . The average ages at Weaning, On, Off, and Finish were 22.9, $72.7,152.4$, and $174.3 \mathrm{~d}$, respectively. The ranges in the stages of Off and Finish overlapped due to the short time between measurements. The reduced numbers of records for the stages of On, Off, and Finish were not caused by selection and were not an artifact of the population, with the exception of natural death.

\section{Statistical analysis}

Genetic parameters, including variance components and ratios, were estimated using the restricted maximum likelihood (REML) procedure based on an animal model, using the Wombat program (Meyer, 2010) which is able to accommodate various regression in a mixed model equation for covariates. The statistical model for the traits was as follows.

$$
\mathrm{y}_{\mathrm{i}}=\mathrm{Xb}_{\mathrm{i}}+\mathrm{Za}_{\mathrm{i}}+\mathrm{Wm}_{\mathrm{i}}+\mathrm{e}_{\mathrm{i}}
$$

where $y_{\mathrm{i}}$ is the vector of observations for the $i$ th trait, and $b_{i}$ is the vector of fixed effects, including the contemporary group, birth year-month, sex, sow parity, and covariate age at measurement (except birth weight). For random effects, $a_{i}$ is the vector of random animal effects, $m_{i}$ is the vector of correlated random maternal genetic effects, and $e_{i}$ is the vector of random residual effects. $\mathrm{X}, \mathrm{Z}$, and $\mathrm{W}$ are incidence matrices relating records of a trait to fixed effects and random effects.

If $\mathrm{a}$ and $\mathrm{m}$ represent the vectors of direct animal genetic effects and maternal genetic effects for 5 body weights, and e represents the vector of residual effects, then the random effects can be assumed to follow a normal distribution with zero mean and the following distribution parameters :

$$
\operatorname{var}\left[\begin{array}{c}
\mathrm{a} \\
\mathrm{m} \\
\mathrm{e}
\end{array}\right]=\left[\begin{array}{ccc}
\mathrm{A} \otimes \mathrm{G}_{\mathrm{d}} & 0 & 0 \\
0 & \mathrm{~A} \otimes \mathrm{G}_{\mathrm{m}} & 0 \\
0 & 0 & \mathrm{I} \otimes \mathrm{R}
\end{array}\right]
$$

where $A$ is the numerator relationship matrix; $G_{d}$ is a matrix with order 5 , the covariance matrix of the direct genetic effect between traits; and $G_{m}$ is the covariance matrix of maternal genetic effects. Matrix I is the identity matrix of appropriate dimension in each case, and $\mathrm{R}$ is the covariance matrix of residual effect.

Ordinary polynomials (POL) and Legendre polynomials

\begin{tabular}{|c|c|c|c|c|c|c|c|c|c|}
\hline \multirow{2}{*}{ Stage $^{1}$} & \multirow{2}{*}{$\mathrm{N}$} & \multicolumn{4}{|c|}{ Body weight (kg) } & \multicolumn{4}{|c|}{ Age $(\mathbf{d})$ at measurement } \\
\hline & & Mean & SD & Min. & Max. & Mean & SD & Min. & Max. \\
\hline Birth & 5,064 & 1.39 & 0.28 & 0.64 & 2.59 & & & & \\
\hline Weaning & 5,071 & 6.4 & 1.5 & 1.2 & 12.5 & 22.9 & 3.4 & 17 & 34 \\
\hline On & 4,953 & 25.7 & 5.9 & 6 & 48 & 72.7 & 4.8 & 51 & 88 \\
\hline Off & 2,836 & 89.4 & 10.0 & 50 & 127 & 152.4 & 7.8 & 124 & 165 \\
\hline Finish & 1,186 & 102.8 & 12.0 & 38 & 140 & 174.3 & 8.5 & 152 & 218 \\
\hline
\end{tabular}

Table 2. Basic statistics for the body weights and ages at measurement

${ }^{1}$ Birth = Birth; Weaning = Weaning; On = The beginning of performance test; Off $=$ The end of performance test; Finish = Harvest. 
(LEG: Bayin, 2006) with order 1, 2, and 3 were adopted to adjust body weight with age at Weaning, On, Off, and Finish. The fitting of the polynomial models was checked with Information Criterion of Akaike (AIC; Akaike, 1973), and the prediction error deviation (PED) was described as follows.

$$
\mathrm{PED}=\frac{1}{\mathrm{n}} \sqrt{\sum\left(\hat{\mathrm{w}}_{\mathrm{i}}-\overline{\mathrm{w}}_{\mathrm{i}}\right)^{2}}
$$

where $\hat{\mathrm{W}}_{\mathrm{i}}$ and $\overline{\mathrm{W}}_{\mathrm{i}}$ are the predicted weight at age $\mathrm{i}$ by polynomial regressions and average weight at age $\mathrm{i}$, respectively. Further, $\mathrm{n}$ is the number of predicted weights at a stage or all stages.

\section{RESULTS AND DISCUSSION}

This study aimed to examine the models to characterize pre- and post-weaning pig growth up to harvest in the context of direct and maternal genetic effects on body weight. The large standard errors obtained by Tomiyama et al. (2010) were possibly due to the relatively small data set of Berkshire pigs used in their study (Meyer, 1992). Further, trying to estimate too many parameters in a model may result in inaccurate estimations (Tomiyama et al., 2010) The correlations of direct and maternal genetic effects with body weight yielded standard errors within the range of 0.35 to 0.79 in the preliminary analysis in the present study, which yielded inaccurate estimates of other genetic parameters due to missing data in the multivariate analysis (Kachman and Van Vleck, 2007) and possibly low number of dams with records (141, as shown in Table 1). Therefore, the covariance for direct and maternal genetic effects was assumed to be null in this study.

Cross-fostering was not needed very often in the herd, since there were 7.6 piglets per litter in the Berkshire herd in this study; hence, confounding of direct and maternal genetic effects rarely occurred (Meyer, 1992). Despite the importance of including litter effects in the model (Satoh et al., 2002), common environmental effects within the same litter due to dam were not applicable to growth after weaning. Uncorrelated maternal effects of the foster dam or common environmental effects were not taken into account. The maternal genetic effects on growth in the models, however, were roughly assumed to include genetic effects and fostering ability of the dam.

Significance levels of fixed effects and covariates were checked by the GLM procedure of SAS. The effects of birth year-month, sex, and sow parity on body weight, except sex effect on body weight at weaning, were highly significant ( $p$ $<0.01)$. The sex effects at weaning, however, were included in the multivariate analysis model. Males were heavier than females at birth (1.47 to $1.42 \mathrm{~kg}$ ) and at weaning (6.55 to $6.48 \mathrm{~kg}$ ), and males grew faster during the test period (Table 3). Castrates had similar body weight as males at weaning $(6.55 \mathrm{~kg})$ and exhibited the fastest growth during the postweaning period (least square means were 88.5, 92.2, and $92.6 \mathrm{~kg}$ for females, males, and castrates at Off and 100.1, 103.4 , and $107.5 \mathrm{~kg}$ at Finish, respectively). The ancestors of the Berkshire pigs used in the present study were imported from the United States, and they grew faster than the Japanese Berkshire pigs; they were aged $210 \mathrm{~d}$ at harvest (Tomiyama et al., 2009). Rosendo et al. (2007) and Carney et al. (2009) mentioned that sow parity affects early growth but has no effects on performance test traits. Firstlitter piglets, however, without exception in this study were lighter at Birth, Weaning, On, Off, and Finish (1.36, 5.97, $24.8,90.7$, and $103.5 \mathrm{~kg}$, respectively; Table 3) than pigs of any other parity.

\section{Adjustment of body weight with age}

Since the test pigs were not weighed on a precise date, age adjustment for body weight development is important for estimating genetic parameters and evaluating genetic superiority of the animal. The logistic model is the best fit for the characteristic of body development in pigs and increased weight gains after 7 wks resulted in a sigmoid growth curve for Göttingen minipigs (Köhn et al., 2007). However, it is difficult to fit the entire lifespan of a pig with a model on the basis of longitudinal body weight records. Age was a covariate in the multivariate model for the longitudinal body weight records, which were usually adjusted with pre-calculated coefficients of age for the performance test. Table 3 shows the prediction error deviation (PED) and Information Criterion of Akaike (AIC)

Table 3. Least square means of body weights $(\mathrm{kg})$ according to sex and sow parity

\begin{tabular}{|c|c|c|c|c|c|c|c|c|}
\hline \multirow{2}{*}{ Stage $^{1}$} & \multicolumn{3}{|c|}{ Sex } & \multicolumn{5}{|c|}{ Sow parity } \\
\hline & Female & Male & Castrate & 1 & 2 & 3 & 4 & $\geq 5$ \\
\hline Birth & 1.42 & 1.47 & 1.46 & 1.36 & 1.48 & 1.45 & 1.50 & 1.47 \\
\hline Weaning & 6.48 & 6.55 & 6.55 & 5.97 & 6.68 & 6.78 & 6.61 & 6.59 \\
\hline On & 25.7 & 26.1 & 26.1 & 24.8 & 25.1 & 26.9 & 26.6 & 26.4 \\
\hline Off & 88.5 & 92.2 & 92.6 & 90.7 & 91.3 & 90.8 & 91.4 & 91.3 \\
\hline Finish & 100.1 & 103.4 & 107.5 & 102.5 & 105.6 & 103.5 & 103.3 & 103.6 \\
\hline
\end{tabular}

${ }^{1}$ On $=$ The beginning of performance test; Off $=$ The end of performance test ; Finish = Harvest . 


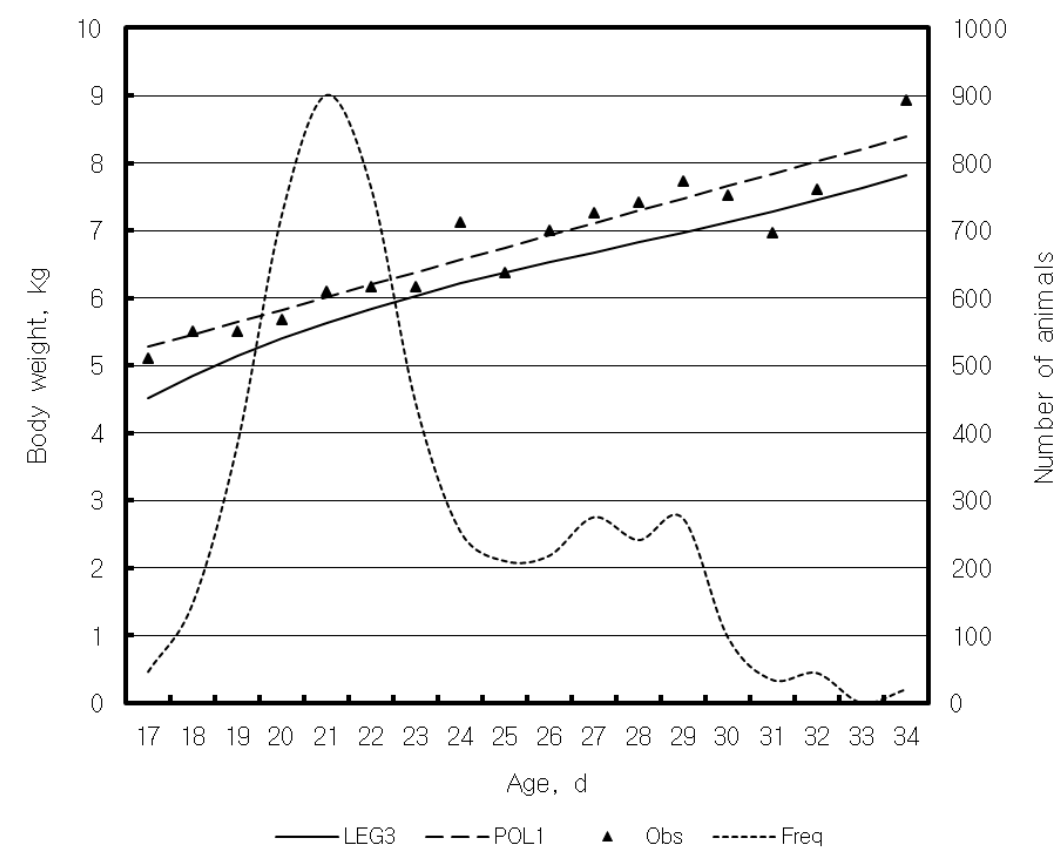

Figure 1. Prediction of Legendre 3rd polynomial (LEG3) and 1st order polynomial (POL1) and the frequency of records for body weight at weaning (Weaning).

according to the polynomial models for age. Although LEG3 is fitted best by AIC and overall PED, it did not yield the minimum PED for each body weight in the multivariate analysis for the body weights at each stage. The 1 st order polynomial model (POL1), however, fitted quite well with each stage of life (least PED for the body weights at Weaning and On, 2nd least PED for the body weights at Off and Finish) and also produced a small overall PED (6.69). Linear regression (POL1) is characterized by least prediction error (Rohatgi, 1976), which has the same property as the minimum PED. Figure 1 and 2 show traces of prediction by polynomials, observed means, and frequency of records in the stage of Weaning and Off. POL1 fit better with the observed weight means at Weaning in Figure 1 and produced a smaller PED than LEG3 in Table 4. The sigmoid curve by POL3 fit best with Off within the range of 124 to $165 \mathrm{~d}$ in Figure 2, which had a similar shape as that obtained with the logistic model (Köhn et al., 2007).

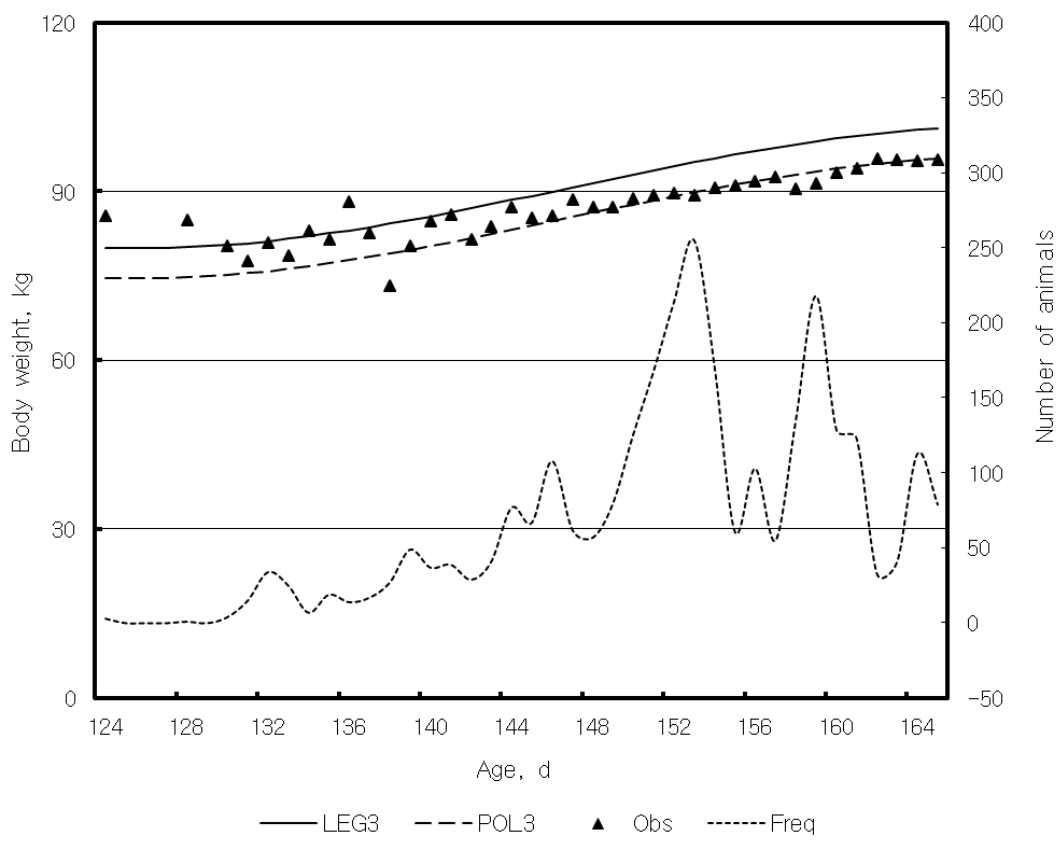

Figure 2. Prediction of Legendre 3rd polynomial (LEG3), ordinary 3rd polynomial (POL3), and the frequency of records for body weight at the end of the performance test (Off). 
Table 4. The information criteria of Akaike and prediction error deviation according to polynomial models used to adjust age (d) on body weight

\begin{tabular}{|c|c|c|c|c|c|c|c|}
\hline \multirow{2}{*}{ Models } & \multirow{2}{*}{ Order } & \multicolumn{4}{|c|}{ Prediction error deviation (PED) } & \multirow{2}{*}{ Overall PED } & \multirow{2}{*}{$\mathrm{AIC}^{2}$} \\
\hline & & Weaning $^{1}$ & On & Off & Finish & & \\
\hline \multirow{3}{*}{$\begin{array}{l}\text { Ordinary } \\
\text { polynomial }\end{array}$} & 1 & 0.34 & 2.18 & 4.13 & 9.98 & 6.69 & 35,647 \\
\hline & 2 & 0.37 & 3.55 & 4.47 & 19.28 & 12.17 & 35,677 \\
\hline & 3 & 0.35 & 3.26 & 3.97 & 19.22 & 12.10 & 35,730 \\
\hline \multirow{3}{*}{$\begin{array}{l}\text { Legendre } \\
\text { polynomial }\end{array}$} & 1 & 0.55 & 4.04 & 5.21 & 18.88 & 12.05 & 38,519 \\
\hline & 2 & 0.57 & 3.84 & 5.44 & 18.73 & 11.98 & 35,615 \\
\hline & 3 & 0.55 & 3.43 & 4.80 & 9.59 & 6.64 & 35,612 \\
\hline
\end{tabular}

${ }^{1}$ On $=$ The beginning of performance test; Off = The end of performance test; Finish = Harvest.

${ }^{2} \mathrm{AIC}=$ Information Criteria of Akaike.

Scaling effect was indicated by the increase in standard deviation with increased mean body weight at Finish. Consequently, the stage that influenced the fitness of the model the most was Finish. LEG3 fit best in the Finish stage with concerning PED and subsequently yielded the lowest overall PED and AIC. We may conclude that the accuracy of adjustment by age for body weights should not rely only on the AIC of polynomial models, at least for multivariate analysis of longitudinal body weights. The result, however, is not conclusive because in the estimated genetic parameters, no considerable tendency and differences were discovered between POL1 and LEG3. To obtain a clear conclusion, further research that includes a simulation study may be required. Alternatively, growth itself can be analyzed using random regression models.

\section{Environmental and phenotypic correlations}

Table 5 and 6 show direct and maternal genetic heritabilities and their correlations with body weights at different stages and environmental and phenotypic correlations, estimated using REML with LEG3. The environmental correlations were estimated from the variance and covariance components of residual effects, which included permanent and temporary environmental effects and error. With the assumption of no covariance between errors, it is possible to estimate environmental correlations with residual (co)variance, including both permanent and temporary environment effects. Body weights of pigs at different growth stages are positively associated each other (Mahan and Lepine, 1991; Main et al., 2004; Alison et al., 2007; Fix et al., 2010), which were consistent with the positive phenotypic and environmental correlations summarized in Table 5 and 6 . The estimated environmental correlation between body weight at Birth and Weaning was moderately low (0.39), but correlations between body weight at Birth and at On, Off, and Finish were fairly weak $(0.23,0.27$, and 0.29 , respectively; Table 6). The correlations between body weight at Weaning and at On, Off, and Finish continuously decreased $(0.39,0.41$, and 0.37 , respectively). The correlations between body weight at On and at Off and Finish were moderate (0.32 and 0.37, respectively), whereas the correlation between body weight at Off and Finish was as strong as 0.78. The standard errors of environmental correlation were low, within the range of 0.02 to 0.08 . Piglets stayed with the sow during the suckling period. They mingled with other litters in the pens after weaning. Large pens were used to accommodate the pigs as they grew. After the suckling period, pen mates could be changed until Finish. The approximate time intervals between measurements were $22 \mathrm{~d}, 50 \mathrm{~d}, 80 \mathrm{~d}$, and $22 \mathrm{~d}$ between Birth and Weaning, Weaning and On, On and Off, and Off and Finish, respectively (Table 1). The environmental correlations may reflect farm management and interval between the measurements. The environmental correlation between body weight at Off and at Finish was stronger $(0.78)$ than any other environmental correlation

Table 5. Estimates ${ }^{1}( \pm \mathrm{SE})$ of direct genetic heritabilities and correlations and phenotypic correlations on body weights are along the diagonal, upper diagonal, and lower diagonal, respectively, and phenotypic $\operatorname{SD}\left(\sigma_{\mathrm{P}}\right)$ is at the bottom

\begin{tabular}{lllllc}
\hline Stage $^{2}$ & Birth & Weaning & On & Off & Finish \\
\hline Birth & $0.09 \pm 0.03$ & $0.48 \pm 0.22$ & $0.36 \pm 0.22$ & $0.10 \pm 0.23$ & $0.10 \pm 0.24$ \\
Weaning & $0.40 \pm 0.03$ & $0.11 \pm 0.04$ & $0.33 \pm 0.20$ & $0.25 \pm 0.20$ & $0.17 \pm 0.21$ \\
On & $0.27 \pm 0.02$ & $0.42 \pm 0.02$ & $0.20 \pm 0.05$ & $0.67 \pm 0.11$ & $0.76 \pm 0.10$ \\
Off & $0.27 \pm 0.03$ & $0.37 \pm 0.03$ & $0.47 \pm 0.02$ & $0.31 \pm 0.06$ & $0.92 \pm 0.06$ \\
Finish & $0.24 \pm 0.03$ & $0.32 \pm 0.03$ & $0.51 \pm 0.03$ & $0.83 \pm 0.02$ & $0.43 \pm 0.09$ \\
$\sigma_{\mathrm{P}}$ & 0.27 & 1.39 & 5.15 & 8.89 & 11.28 \\
\hline
\end{tabular}

${ }^{1}$ Obtained by Legendre polynomials with order 3.

${ }^{2}$ Birth $=$ Birth; Weaning $=$ Weaning; On $=$ The beginning of performance test Off $=$ The end of performance test Finish $=$ Harvest. 
Table 6. Estimates ${ }^{1}( \pm \mathrm{SE})$ of maternal genetic heritabilities and correlations and environmental correlations on body weights by Legendre polynomials with order 3 are along the diagonal, upper diagonal, and lower diagonal, respectively

\begin{tabular}{lccccc}
\hline Stage $^{2}$ & Birth & Weaning & On & Off & Finish \\
\hline Birth & $0.27 \pm 0.03$ & $0.40 \pm 0.08$ & $0.37 \pm 0.11$ & $0.54 \pm 0.12$ & $0.39 \pm 0.17$ \\
Weaning & $0.39 \pm 0.02$ & $0.34 \pm 0.03$ & $0.59 \pm 0.08$ & $0.49 \pm 0.11$ & $0.49 \pm 0.16$ \\
On & $0.23 \pm 0.03$ & $0.39 \pm 0.03$ & $0.15 \pm 0.03$ & $0.88 \pm 0.08$ & $0.65 \pm 0.14$ \\
Off & $0.27 \pm 0.04$ & $0.41 \pm 0.04$ & $0.32 \pm 0.04$ & $0.10 \pm 0.03$ & $0.90 \pm 0.09$ \\
Finish & $0.29 \pm 0.05$ & $0.37 \pm 0.05$ & $0.37 \pm 0.05$ & $0.78 \pm 0.08$ & $0.10 \pm 0.04$ \\
\hline
\end{tabular}

${ }^{1}$ Obtained by Legendre polynomials with order 3.

${ }^{2} \mathrm{On}=$ The beginning of performance test Off $=$ The end of performance test Finish = Harvest.

because pen mates had to stay together due to a small separation time $(22 \mathrm{~d})$ between Off and Finish. The environmental correlation of body weight at Birth with that at Weaning was moderate $(0.39)$, which may be explained by the common environmental effects on all pigs in a litter fostered by the same sow during the suckling period. After weaning, moderate correlations were maintained until Off. The environmental correlation between a pair of measurements generally decreased as the interval between measurements increased. Phenotypic correlations showed the same tendency as environmental correlations (Table 5). It shows that the phenotypic relationship among body weights at different stages approximately corresponds to environmental relationship, even though direct and maternal genetic correlations were apparently different with phenotypic and environmental correlations (Tables 5 and 6).

\section{Direct and maternal genetic correlations}

The correlations of direct genetic effects on body weight were positive without exception and gradually weakened as the interval between measurements increased. The direct genetic correlations of body weight at Birth and other body weights were $0.48,0.36,0.10$, and 0.10 (Weaning, On, Off, and Finish, respectively; Table 5). Similar phenomena appeared in the correlation between the body weight at Weaning and other body weights. The only exception was the direct genetic correlation between body weight at On with body weight at Off (0.67), which was weaker than the correlation with body weight at Finish (0.76). The direct genetic correlations of body weight at Birth with other body weights and that at Weaning with other body weights were weaker than the results by Tomiyama et al. (2010) and had relatively large standard errors within the range of 0.20 to 0.24 due to missing data in the multivariate analysis (Kachman and Van Vleck, 2007), which were not significantly different from null correlations. This finding, however, suggests that birth and weaning weight have a positive direct genetic relationship with the body weights in the later stages (Off and Finish).

All estimates of maternal genetic correlation were positive. The estimated maternal genetic correlations between body weight at Finish and other body weights were
0.39, 0.49, 0.65, and 0.90 (Birth, Weaning, On, and Off, respectively; Table 6). The maternal genetic correlations of body weight at Birth with Weaning, On and Off were 0.40, 0.37 , and 0.54 , respectively. The maternal genetic correlations of body weight at Weaning with body weights at On and Off were 0.59 and 0.49 , respectively. The estimated maternal correlations were noticeably weaker and the estimated standards errors of the correlations were relatively smaller than the estimates reported by Tomiyama et al. (2010), possibly because of the assumption of null direct and maternal genetic covariances. The correlations of maternal genetic effects had standard errors within the range of 0.08 to 0.17 , which were smaller than those of the direct genetic correlations. With an increase in the time intervals between measurements, the estimated correlations gradually decreased except the correlations of body weight at Birth. The direct genetic correlations exhibited the same tendency, with exception of the correlations of body weight at On with body weights at Finish. Comparing with the direct genetic correlations, the maternal genetic correlations were generally large even when the interval between measurements was large. Consistent genetic relations among prenatal growth, mothering ability, and nonchromosomal genetic effects on body weight development possibly lead to stronger positive maternal genetic correlations with smaller standard errors than those with direct genetic correlations. Furthermore, significantly weaker positive associations between pre- (Birth and Weaning) and post- (On, Off and Finish) weaning traits, were shown in the direct genetic effects comparing with the maternal genetic effects. It indicates that the genetic effects of individuals over body weights at different stages were not influential as much as maternal genetic effects. The results are not conclusive because the genetic correlations between direct and maternal genetic effects are excluded in the model of the present study. Conflicting results on the direct and maternal genetic correlations of body weights at early stages reported by Solanes et al. (2004) and Tomiyama et al. (2010), however, showed a positive relationship among body weights at different stages in maternal genetic effects. It suggests that selection on maternal genetic effects at the early developmental stage of 
pigs could be an effective choice for genetic improvement of body weights.

\section{Heritabilities}

Variance components were estimated with relatively small standard errors (only phenotypic standard deviations are presented, as seen in Table 5). The estimated heritabilities of the direct genetic effect progressively increased from birth to harvest and were low for body weight at Birth, Weaning, and On (0.09, 0.11, and 0.20, respectively), moderate at Off (0.31), and high for body weight at Finish (0.43; Table 5). The estimated heritabilities of the maternal genetic effect decreased and were moderate for body weight at Birth (0.27) and Weaning (0.34) and low for body weight at On, Off, and Finish $(0.15,0.10$, and 0.10 , respectively; Table 6). The estimates of SE for both direct and maternal genetic heritabilities were small, the range of which was from 0.03 to 0.09 (Tables 5 and 6). The direct genetic heritabilities for body weight at Birth, Weaning, and On were not highly heritable compared with the maternal genetic heritabilities for the body weights in the early stages. On the other hand, the estimated direct heritabilities for body weight in the later stages of Off and Finish were high and the maternal genetic heritabilities were as low as 0.10 for body weight at both stages.

The direct genetic heritability increased up to body weight at Finish, whereas the heritability of the maternal genetic effect decreased during the same period, the exception being for body weight at Weaning ( 0.27 for Birth, 0.34 for Weaning, and 0.15 for On). As pigs age, maternal genetic influences on pigs are reduced and pigs rely more on their own gene expression.

The direct genetic heritabilities of body weights at birth, weaning, and at $60 \mathrm{~d}$ of age for Berkshire pigs were 0.07 , 0.14 , and 0.18 , respectively (Tomiyama et al., 2010), which are similar to those reported in this study. However, the tendency of these estimates of maternal genetic heritability for body weights in the early stages (Birth, Weaning, and On) is not consistent with previous reports (Tomiyama et al., 2010). The maternal heritabilities reported by Tomiyama et al. (2010) were $0.19,0.06$, and 0.03 , respectively, which were smaller than those $(0.27,0.34$, and 0.15 , respectively) reported in this study. This difference is probably because common environmental effects were excluded and null covariances of direct and maternal genetic effects were assumed in the model. The estimates from data with maternal pedigree from a closed population in this study were also possibly a cause for discrepancy. However, the maternal genetic heritabilities of birth and weaning weight by Rosendo et al. (2007) were 0.17 and 0.32 , respectively, which are closer to the estimates reported in this study. The influences of maternal genetic effects on prenatal growth and mothering ability were no longer valid after weaning.
However, maternal genetic heritabilities for the body weights after weaning were low, but there remained evidence of existing maternal genetic influence.

The results suggest that the body weight of younger pigs is more influenced by maternal gene expression, which may include maternal genetic effects of prenatal growth and mothering capability and possibly a small influence of nonnuclear genetic effects such as mitochondrial genetic effects (Margit et al., 1963). Thus, the estimated maternal genetic effect on body weight of progeny at Off and Finish must be added in the sow's superiority. In conclusion, although maternal genetic effects may be weak (low maternal genetic heritabilities), they are sustained through life (relatively high maternal genetic correlations).

\section{ACKNOWLEDGEMENT}

This work was supported by the 2010 sabbatical year research grant of the University of Seoul.

\section{REFERENCES}

Akaike, H. 1973. Information theory and an extension of the maximum likelihood principle. 2nd Int. Symp. Information Therory (Ed. B. N. Petrov and F Csaki) Akademiai Kiado, Budapest, Hungary.

Bayin, S. S. 2006. Mathematical Methods in Science and Engineering, Wiley, Chapter 2.

Carney, E. E., H. Tran, J. W. Bundy, R. Moreno, P. S. Miller and T. E. Bu rky. 2009. Effect of dam parity on growth performance and immunity of weaned pigs. Nebraska Swine Reports. 2009:29-32.

Coffey, R. D., G. R. Parker and K. M. Laurent. 1995. Feeding growing-finishing pigs to maximize lean growth rate. University of Kentucky, Lexington, and Kentucky State University, Frankfort. Cooperative Extension Service. ASC147.

Do, C. H. 2007. Estimation of growth traits using growth curve in Gyungnam-heugdon (Berkshire). J. Anim. Sci. Technol. (Korea) 49:195-202.

Fix, J. S., J. P. Cassady, J. W. Holl, W. O. Herring, M. S. Culbertson and M. T. See. 2010. Effect of birth weight on survival and quality of commercial market swine. Livest. Sci. 132:98-106.

Kachman, S. D. and L. D. Van Vleck. 2007. Technical Note: Calculation of standard errors of estimates of genetic parameters with the multiple-trait derivative-free restricted maximal likelihood programs. J. Anim. Sci. 85:2375-2381.

Kim, H. S., B. W. Kim, H. Y. Kim, H. T. Iim, H. S. Yang, J. I. Lee, Y. K. Joo, C. H. Do, S. T. Joo, J. T. Jeon and J. G. Lee. 2007. Estimation of terminal sire effect on swine growth and meat quality traits. J. Anim. Sci. Technol. (Korea) 49:161-170.

Köhn, A. F., R. Sharifi and H. Simianer. 2007. Modeling the growth of the Goettingen minipig. J. Anim. Sci. 85:84-92.

Mahan, D. C. and A. J. Lepine. 1991. Effect of pig weaning weight and associated nursery feeding programs on subsequent performance to 105 kilograms body weight. J. Anim. Sci. 
69:1370-1378.

Main, R. G., S. S. Dritz, M. D. Tokach, R. D. Goodband and J. L. Nelssen. 2004. Increasing weaning age improve pig performance in a multisite production system. J. Anim. Sci. 82:1499-1507.

Meyer, K. 1992. Bias and sampling covariances of estimates of variance components due to maternal effects. Genet. Sel. Evol. 24:487-509.

Meyer, K. 2010. WOMBAT: A program for mixed model analyses by restricted maximum likelihood. http://didgeridoo.une.edu.au/ $\mathrm{km} / \mathrm{homepage.php}$

Margit, M., K. Nass and S. Nass. 1963. Intramitochondrial fibers with DNA characteristics I. Fixation and electron staining reactions. J. Cell Biol. 19:593-611.

Rohatgi, V. K. 1976. An introduction to probability theory and mathematical statistics. John Wiley and Sons. New York. Chapter 8.

Rosendo, A., L. Canario, T. Druet, J. Gogue and J. P. Bidanel. 2007. Correlated responses of pre- and post- weaning growth and backfat thickness to six generations of selection for ovulation rate or prenatal survival in French Large White pigs. J. Anim. Sci. 85:3209-3217.
Satoh, M., C. Hick, K. Ishii and T. Frukawa. 2002. Choice of statistical model for estimating genetic parameters using restricted maximum likelihood in swine. J. Anim. Breed. Genet. 119:285-296

Solanes, F. X., K. Grandinson, L. Rydhmer, S. Stern, K. Andersson and N. Lundeheim. 2004. Direct and maternal influences on the early growth, fattening performance, and carcass traits of pigs. Livest. Prod. Sci. 88:199-212.

Smith, A. L., K. J. Stalder, T. V. Serenius, T. J. Baas and J. W. Mabry. 2007. Effect of piglet birth weight on weights at weaning and 42 days post weaning. J. Swine Health Prod. 15:213-218.

Tomiyama, M., T. Oikawa, M. A. Hoque, T. Kanetani and H. Mori. 2009. Influence of early postweaning traits on genetic improvement of meat productivity in purebred Berkshire pigs. J. Anim. Sci. 87:1613-1619.

Tomiyama, M., T. Kanetani, Y. Tatsukawa, H. Mori and T. Oikawa. 2010. Genetic parameters for preweaning and early growth traits in Berkshire pigs when creep feeding is used. J. Anim. Sci. 88:879-884.

Willham, R. L. 1972. The role of maternal effects in animal breeding: III. Biometrical aspects of maternal effects in animals. J. Anim. Sci. 35:1288-1293. 